\title{
Contrast Enhancement of Multi-Displays Using Human Contrast Sensitivity
}

\begin{abstract}
Study of contrast sensitivity of the human eye shows that we are more sensitive to brightness differences at low intensity levels than at high intensity levels. We apply this fact effectively to achieve brightness seamlessness in multi-projector displays. Multi-displays, popularly made of a rectangular array of partially overlapping projectors, show severe spatial variation in brightness. Existing methods achieve brightness uniformity across the display by matching the brightness response of every pixel to the pixel with the most limited contrast leading to severe compression in the contrast of the display. In this paper, we propose a method that allows a constrained variation in brightness guided by the human contrast sensitivity function such that it is imperceptible to the human eye and this achieves seamless multidisplay. At the same time, such a constrained smoothing of the brightness leads to dramatic improvement in the contrast of the display making it practically usable.
\end{abstract}

\section{Introduction}

The human sensitivity to spatially varying contrast is a wellstudied problem in the perception literature. Contrast sensitivity defines the amount of minimum contrast required for human detection of a spatially varying brightness pattern and shows that the humans are more sensitive to brightness differences at low intensity than at high intensity [29]. In this paper, we use this perceptual phenomenon to achieve brightness seamlessness of large high-resolution displays made by tiling multiple commodity display devices. Such multi-displays offer an inexpensive way to display highresolution life-size images essential for large scale visualization and virtual reality applications used for training, simulation and entertainment. Multi-displays can be built by a rectangular array of LCD panels or projectors. LCD panels cannot provide a physically seamless display due to the presence of visibly thick mullions between adjacent LCD panels. Projectors, on the other hand, can display in a space disassociated from the physical device and hence, can be tiled in a physically seamless fashion. This makes multiprojector displays a popular choice for multi-displays.

In building multi-projector displays there exists several issues such as driving architecture and data distribution [26, 13, 2, 11, 12], but the most important issues to be addressed to make multi-projector display look like a sin- gle display are geometric misalignment and color variation. Several algorithms achieve geometrically undistorted and aligned displays $[24,23,31,10,25,7,5]$. But color variation is still a significant problem.

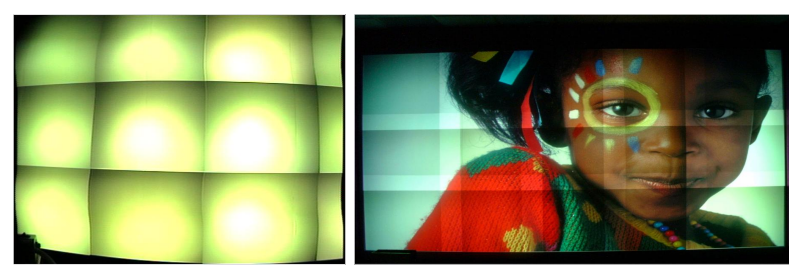

Figure 1: Digital photographs of tiled displays showing the color variation problem. Left: Example of severe color variation across a display made of abutting projectors when every pixel of this display is projecting the identical input of the maximum intensity for green. Right: A tiled display made of a $3 \times 5$ array of fifteen projectors $\left(10^{\prime} \times 8^{\prime}\right.$ in size $)$ with perfect geometric registration, but with color variation.

The spatial color variation in a multi-projector display can be severe (Figure 1) with many factors contributing to it. The most salient are commodity optics of projectors causing a center-to-fringe fall-off in brightness (commonly called the hot-spot effect) pronounced by distance attenuation of light and non-Lambertian screens, variation in age of bulbs and properties of filters across different projectors causing difference in color across the projectors, and partial overlaps across different projectors causing higher brightness overlap regions.

Color is defined by one dimensional brightness and two dimensional chrominance (defining hue and saturation). $[20,16]$ shows that most current multi-displays made of projectors of the same model show large spatial variation in brightness while the chrominance is almost constant. Also, humans are an order of magnitude more sensitive to brightness variation than to chrominance variation $[6,8,29]$. Thus, spatial brightness variation is much more significant than the chrominance variation.

Initial methods of color compensation used blending or feathering techniques to smooth the brightness transition across the overlaps using either software [24], or physical masks mounted at the projector boundaries [15], or optical masks inserted in the light path of the projectors [4]. [21] replaced the different bulbs of the projectors by a single common bulb from which the light was distributed to the different projection devices via optical fibres to remove the color variation introduced by varying bulb ages across different 

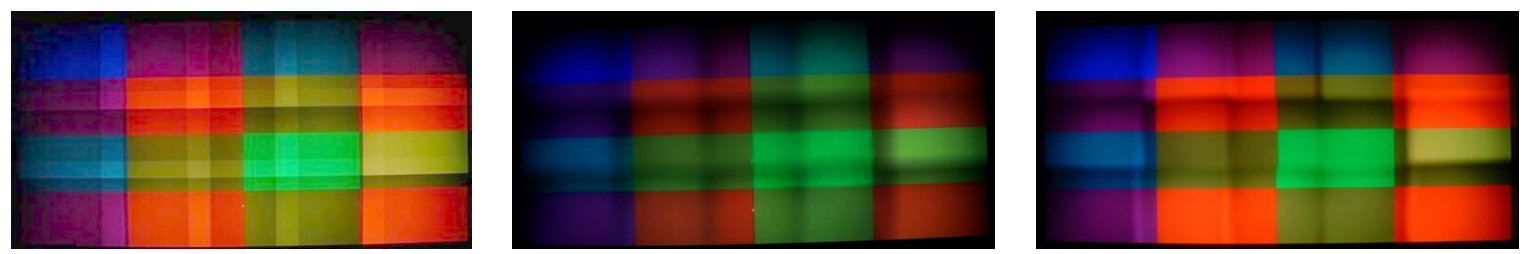

Figure 2: The fifteen projector tiled display before blending (left), after software blending (middle), and after optical blending(right).
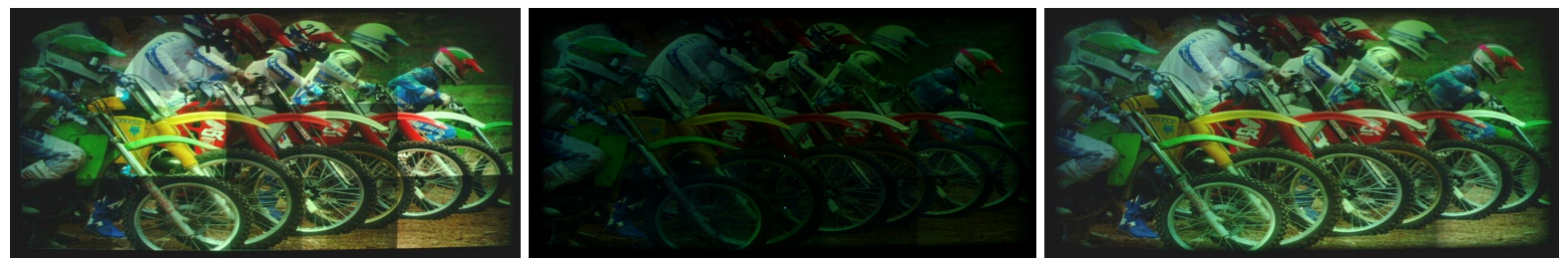

Figure 3: The $10^{\prime} \times 8^{\prime}$ tiled display made of $3 \times 5$ array of four projectors. Left: Before any correction. Middle: After matching the brightness response at every pixel with the pixel having the most limited contrast. Right: Contrast enhancement after smoothing the brightness response.

projectors. However, these methods did not measure the color variation and assumed linear gamma for projectors. Hence, they resulted in softening of the seams in the overlapping region, rather then removing them (Figure 2). The next generation methods no color variation within a projector and used low resolution sensors like a photometer or a spectroradiometer to estimate the color response at one spatial location per projector and then apply a gamut/brightness matching across different projectors [28, 27, 3, 17, 30].

More recently, [19, 18] used a commodity digital camera to capture the spatial brightness variation of a multiprojector display accurately, both across and within the projectors. This variation was then removed by matching the brightness response of every pixel of the display with the pixel with most limited contrast and brightness. This lead to severe degradation in the display quality (Figure 3).

The nature of human contrast sensitivity shows higher sensitivity to brightness differences at low intensity levels than at high intensity levels. Using this fact, we show that instead of achieving an uniform brightness response at every pixel of the display, we can allow the brightness to vary across the display in a smooth manner such that it stays within the sensitivity levels of the human eye and thus is not noticeable or perceptible. In fact, we pose this as an optimization problem where the contrast of the display is maximized while varying the brightness in a constrained fashion, leading to a display that 'looks' seamless, and at the same time, maintains high contrast (Figure 3 and 12). This brightness smoothing is the primary contribution of this paper.

In Section 2, we discuss the use of human contrast sensitivity function in designing our method. Next, we describe our algorithm in details in Section 3 and conclude with future work in Section 4.

\section{Human Sensitivity to Contrast}

In this section, we derive an equation to describe the sensitivity of the human eye to brightness differences at different intensities. The basics of perceptual phenomenon and results that we use here for that purpose, including the figures, are borrowed from [29].
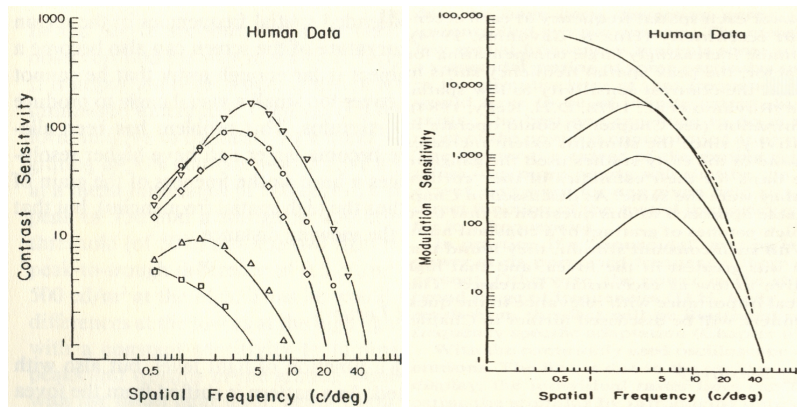

Figure 4: Left: The threshold contrast sensitivity function (CSF) of the human eye. The top most curve is for grating with mean brightness of 5 foot Lamberts. As the mean brightness of the grating decreases, the contrast sensitivity decreases as shown by the lower curves, for 0.5, 0.05, 0.005, and 0.005 foot Lamberts. Right: Absolute modularion sensitivity as a function of brightness level. Here the data from the plot in the left have been replotted to show sensitivity to absolute brightness differences than to contrast (a relative measure). (courtsey [Valois and Valois 1990])

The contrast of a waveform spatially varying in brightness in a sinusoidal fashion is defined as the ratio of its amplitude and mean. Contrast threshold function defines the minimum contrast required to detect such a sinusoidal waveform of a particular mean and spatial frequency. Contrast sensitivity function (CSF) is a reciprocal of the contrast threshold function (Figure 4).

Note that contrast is a relative measure. So, at mean brightness of $1 \mathrm{~cd} / \mathrm{m}^{2}$, a sinusoidal grating of $50 \%$ contrast would have a peak of 0.5 and a trough of $1.5 \mathrm{~cd} / \mathrm{m}^{2}$; at a mean of $1000 \mathrm{~cd} / \mathrm{m}^{2}$, a similar $50 \%$ contrast grating would have a peak of $500 \mathrm{~cd} / \mathrm{m}^{2}$ and a trough of $1500 \mathrm{~cd} / \mathrm{m}^{2}$. Thus, an observer with constant contrast threshold of $50 \%$ at both these intensity levels can only detect a peak-to- 
trough brightness of $1000 \mathrm{~cd} / \mathrm{m}^{2}$ or above at high intensity levels, but a difference as small as $1 \mathrm{~cd} / \mathrm{m}^{2}$ at the low intensity levels. Thus, the observer is 1000 times more sensitive to brightness differences at low intensity levels than at high intensity levels. To illustrate this, the threshold CSF is plotted against absolute brightness differences (Figure 4). This shows that at very high spatial frequencies, sensitivity to absolute brightness differences roughly converge to the same curve, but for most of the frequency spectrum, the sensitivity to absolute brightness differences increases as intensity decreases. This can also be described by the Weber Law.

From this fact we derive a simple equation that says that for a spatially varying brightness field $L(x, y)$, if the absolute luminance difference at any point (given by the derivative of the brightness at that point in the direction $x$ ) is within a fraction $\lambda$ of the original brightness, i.e.,

$$
\frac{\partial L}{\partial x} \leq \lambda L
$$

it can go undetected by the human eye. The fraction $\lambda$ is guided by the Weber Law. Note that, as per Equation 1, as the intensity reduces, the absolute brightness difference also reduces indicating higher sensitivity and vice versa, as predicted by the CSF.

\section{Algorithm}

We pose the problem of achieving brightness seamlessness in multi-displays as an optimization problem. We seek to maximize a scalar optimization function capturing the contrast of the display subject to the constraint of Equation 1. First, we formulate the problem and describe the method for gray multi-displays and then extend it to color displays.

\subsection{Gray Multi-Display}

Let us first consider a single projector. The brightness $B(x, y, i)$ at a pixel $(x, y)$ of the projector for an input $i$, $0.0 \leq i \leq 1.0$ is given by

$$
B(x, y, i)=g(i) \cdot H(x, y)
$$

where $g(i)$ is non-linear gamma function and $H(x, y)$ is the maximum brightness that can be projected at pixel $(x, y)(H$ standing for 'highest'). Equation 2 assumes that $g$ does not vary spatially within a projector, that has been empirically verified to be true in [16]. The second simplifying assumption made in Equation 2 is that the brightness projected for black, i.e. $i=0.0$, is zero (no light is projected for the black input). However, it has been shown in many previous work $[17,28,20]$ that projectors project some light even with input $i=0.0$ (commonly called the black offset). Hence, we modify Equation 2 to take account for the black offset as follows,

$$
B(x, y, i)=g(i) \cdot(H(x, y)-L(x, y))+L(x, y)
$$

where $L(x, y)$ is the minimum brightness projected at pixel $(x, y)$ ( $L$ standing for 'lowest'). Also note that, if $g$ is a monotonic function, then the maximum and minimum brightness at $(x, y)$ occurs for input $i=1.0$ and $i=0.0$ respectively. Or, in other words, $H(x, y)=B(x, y, 1)$ and $L(x, y)=B(x, y, 0)$. But, it has been shown in [20] that gamma function for projectors are often non-monotonic. Hence, the maximum and minimum brightness response may not occur for input 1.0 and 0.0 respectively. The functions $H$ and $L$ for a projector are illustrated in Figure 5.
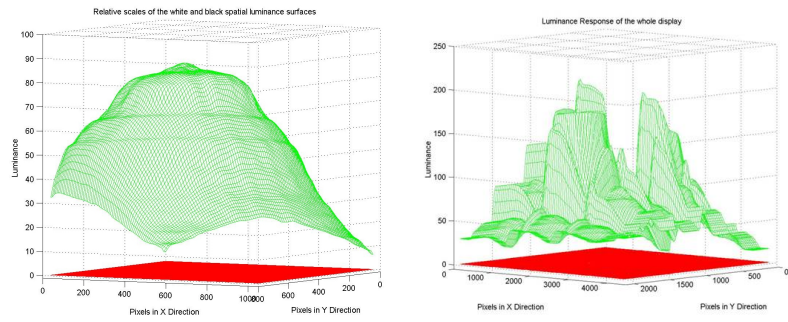

Figure 5: Left: $H(x, y)$ (in green) and $L(x, y)$ (in red) for a single projector. Right: The $H_{D}(x, y)$ and $L_{D}(x, y)$ generated by spatially compositing the $H$ and $L$ from different projectors in a 15 projector display tiled in a $3 \times 5$ array. The high luminance regions in correspond to the overlap regions across different projectors.

Next, we consider a multi-display where one or more projectors can project at a pixel $(x, y)$. Let $j$ projectors contribute at pixel $(x, y), j=1$ indicates that no two projectors overlap at the pixel $(x, y) . j>1$ indicates that multiple projectors overlap at that pixel $(x, y)$. The brightness response $B_{D}(x, y, i)$ at any pixel $(x, y)$ of the multi-display $D$ is the sum of the brightness response of all the projectors contributing to that pixel. Formally, it is written as

$$
\begin{aligned}
B_{D}(x, y, i) & =\sum_{j} B_{j}(x, y, i) \\
& =\sum_{j}\left(g_{j}(i) \cdot\left(H_{j}(x, y)-L_{j}(x, y)\right)+L_{j}(x, y 5)\right.
\end{aligned}
$$

\subsubsection{Brightness Uniformity}

$[20,18]$ presents a method to achieve uniform brightness response at every pixel $(x, y)$ of a multi-display. In this method, a digital camera is used measure the parameters $g$, $H$ and $L$ for every projector. This requires geometric calibration information for which any existing methods can be used [23, 31, 10, 25, 5]. Next, the brightness response at every pixel is matched to achieve uniform brightness throughout the display. In this subsection, we describe this method using the parameters of Equation 5 devised in this paper. This treatise is a formal way to analyze the method presented in $[20,18]$.

First, a uniform gamma function, $G$, is chosen for all projectors reducing Equation 5 to

$$
\begin{aligned}
B_{D}(x, y, i)= & G(i) \cdot\left(\sum_{j} H_{j}(x, y)-\sum_{j} L_{j}(x, y)\right) \\
& +\sum_{j} L_{j}(x, y) \\
= & G(i) \cdot\left(H_{D}(x, y)-L_{D}(x, y)\right)+L_{D}(x, y)(8)
\end{aligned}
$$


where $H_{D}$ and $L_{D}$ are the maximum and minimum brightness response of the whole display and are computed by spatially compositing the $H$ and $L$ from each projector.The $H_{D}$ and $L_{D}$ for a fifteen projector display is illustrated in Figure 5.

To achieve brightness uniformity, the spatial variation in $H_{D}$ and $L_{D}$ is removed by modifying them to $H_{D}^{\prime}$ and $L_{D}^{\prime}$ respectively such that

$$
H_{D}^{\prime}(x, y)=\min _{\forall(x, y)} H_{D}(x, y) ; L_{D}^{\prime}(x, y)=\max _{\forall(x, y)} L_{D}(x, y)
$$

Thus, the brightness response at every pixel is matched to the 'worst' pixel with the most limited contrast and the modified brightness response of the display is given by,

$$
B_{D}(x, y, i)=G(i) .\left(H_{D}^{\prime}(x, y)-L_{D}^{\prime}(x, y)\right)+L_{D}^{\prime}(x, y) .
$$

The $H_{D}$ and $H_{D}^{\prime}$ thus generated for a four projector display is illustrated in Figure 6. However, matching the contrast of all pixels to the pixel with the most limited contrast leads to severe compression in display contrast (Figure 3).

\subsubsection{Brightness Smoothing}

To address this display contrast compression, instead of making $H_{D}^{\prime}\left(L_{D}^{\prime}\right)$ uniform, we allow a variation in them, but constraint in such a manner that it is not perceptible to the human eye. In fact, we pose this problem of modifying $H_{D}\left(L_{D}\right)$ to $H_{D}^{\prime}\left(L_{D}^{\prime}\right)$ as an optimization problem defined by the following optimization constraints.

1. Range Constraint: This constraint of $H_{D}^{\prime}(x, y) \leq$ $H_{D}(x, y)$ ensures that $H_{D}^{\prime}$ never goes beyond the maximum brightness achievable by the display, $H_{D}$. In practice, with discrete sampling of these functions,

$$
H_{D}^{\prime}[x][y]<H_{D}[x][y], \quad \forall x, y .
$$

2. Perceptible Variation Constraint: This constraint is achieved by applying Equation 1 to $H_{D}^{\prime}$ directly as $\frac{\partial H_{D}^{\prime}}{\partial x} \leq \lambda H_{D}^{\prime}$, and assures that $H_{D}^{\prime}$ has a smooth variation imperceptible to humans. $\lambda$ is called the contrast parameter and $\frac{\partial H_{D}^{\prime}}{\partial x}$ is the gradient of $H_{D}^{\prime}$ along any direction $x$. In the discrete domain, when the gradient is expressed as linear filter involving the eight neighbors $\left(x^{\prime}, y^{\prime}\right)$ of a pixel $(x, y), x^{\prime} \in\{x-1, x, x+1\}$ and $y^{\prime} \in\{y-1, y, y+1\}$, this constraint is given by

$$
\frac{\left|H_{D}^{\prime}[x][y]-H_{D}^{\prime}\left[x^{\prime}\right]\left[y^{\prime}\right]\right|}{\sqrt{\left|x-x^{\prime}\right|^{2}+\left|y-y^{\prime}\right|^{2}}} \leq \lambda H_{D}^{\prime}[x][y], \forall x, y, x^{\prime}, y^{\prime} .
$$

3. Contrast Maximizing Objective Function: The above two constraints can yield many feasible $H_{D}^{\prime}$. To maximize contrast, the integration of $H_{D}^{\prime}$ has to be maximized. In discrete domain, this is expressed as

$$
\operatorname{maximize} \sum_{x=0}^{X-1} \sum_{y=0}^{Y-1} H_{D}^{\prime}[x][y]
$$

where $X$ and $Y$ denote the height and width of the multi-display in number of pixels.

We have designed a fast and efficient dynamic programming method that solves this optimization in linear time with respect to the number of pixels in the display i.e. $O(X Y)$. The time taken to compute this solution on Intel Pentium III $2.4 \mathrm{GHz}$ processor for displays with 9 million pixels is less than one second. Figure 6 shows the result of applying the smoothing with different values of $\lambda$ on $H_{D}$ of a four projector display. Note that the special case of $\lambda=0$ corresponds to brightness uniformity. The pseudocode for the algorithm is as follows.

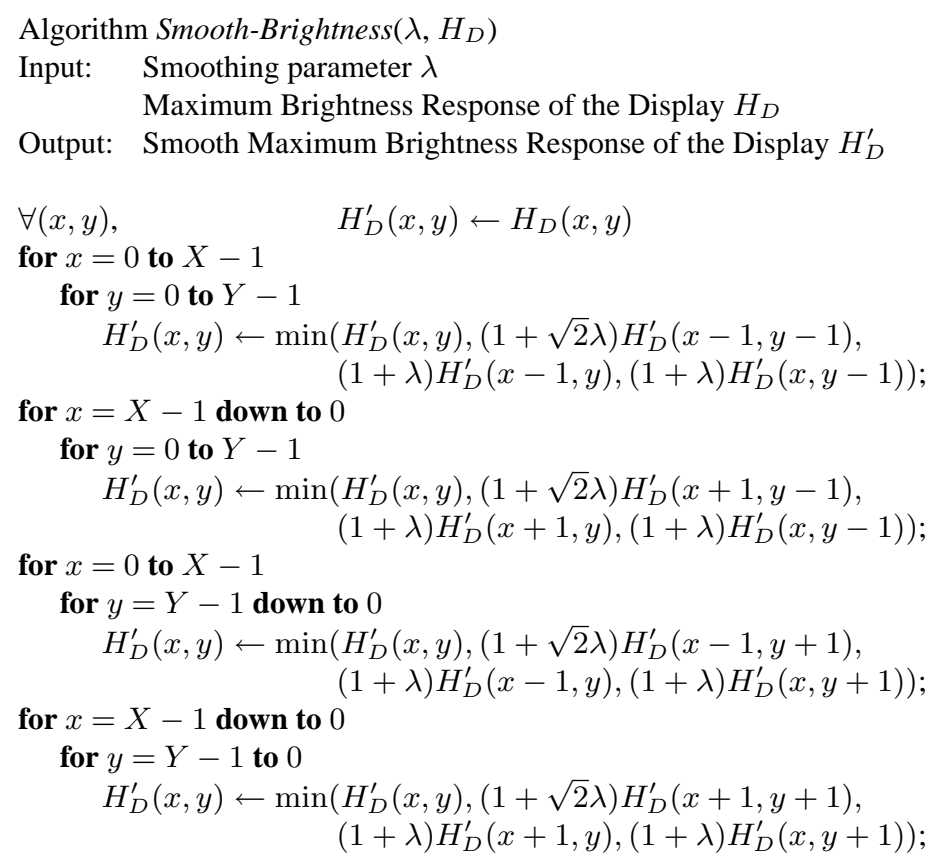

Since $L_{D}$ is a couple of magnitudes smaller than $H_{D}$, applying a similar optimization to $L_{D}$ is an overkill. So, we generate $L_{D}^{\prime}$ as done in [18], given by Equation 9.

The solution to the above optimization problem smooths $H_{D}$ and $L_{D}$ and hence the brightness response $B_{D}$ across the display. The general idea that smoothing the brightness response would achieve the perception of uniformity has been used effectively in the image processing domain in the past $[9,14]$. However, note that the brightness correction for multi-displays cannot be achieved just by smoothing. For example, a popular gradient or curvature based linear smoothing filter will smooth the hills and fill the valleys. However, our constraints are such that while the hills can be smoothed, the valleys cannot be filled since the response thus achieved will be beyond the display capability of the projectors. Hence, the desired smoothing for this particular application was formalized as an optimization problem. 

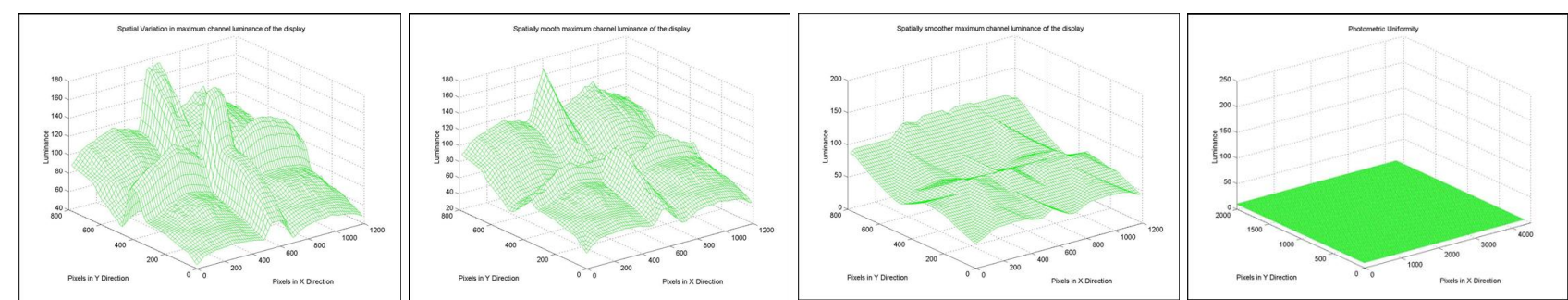

Figure 6: This figure shows the modification of $H_{D}$ for different values of $\lambda$ in a $2 \times 2$ array of four projectors. From left: The original $H_{D}$, the modified $H_{D}^{\prime}$ for $\lambda=0.0025$, for $\lambda=0.00125$ and $\lambda=0 . \lambda=0$ is the special case of brightness uniformity (Section 3.1.1).

\subsubsection{Achieving the Correction}

In the preceding sections we described the process of modifying $H_{D}$ and $g_{i}$ to achieve a seamless display. However, in practice, the projector hardware does not provide us with controls to modify these parameters precisely enough to achieve the effect. So, to achieve the correction we modify the input image to the projector. Similar approaches have been adopted in [20,18], but in this paper we derive this formally from Equation 8.
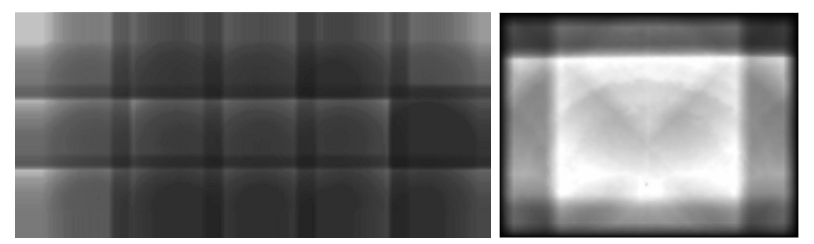

Figure 7: Left: $A(x, y)$, the alpha map for a $5 \times 3$ array of fifteen projectors. Right: The alpha map for a single projector cut out from the $A(x, y)$ of the display.

The actual response of the display at pixel $(x, y)$ for input $i$ is given by Equation 8 and the desired response that we would like to achieve using the modified parameters $G, H_{D}^{\prime}$ and $L_{D}^{\prime}$ is given by Equation 10. To achieve this desired response, we modify the input $i$ to $i^{\prime}$ such that the actual response for input $i^{\prime}$ results in the desired response for input $i$, i.e.

$$
\begin{aligned}
& G\left(i^{\prime}\right) \cdot\left(H_{D}(x, y)-L_{D}(x, y)\right)+L_{D}(x, y) \\
= & G(i) \cdot\left(H_{D}^{\prime}(x, y)-L_{D}^{\prime}(x, y)\right)+L_{D}^{\prime}(x, y)
\end{aligned}
$$

From this we find that,

$$
i^{\prime}=G^{-1}(G(i) \cdot A(x, y)+O(x, y))
$$

where

$A(x, y)=\frac{H_{D}^{\prime}(x, y)-L_{D}^{\prime}(x, y)}{H_{D}(x, y)-L_{D}(x, y)} ; O(x, y)=\frac{L_{D}^{\prime}(x, y)-L_{D}(x, y)}{H_{D}(x, y)-L_{D}(x, y)}$

are called the alpha and offset map for the display. The alpha map represents the per pixel attenuation needed achieve the effect of brightness smoothing and the offset map represents the per pixel offset needed to make the black offset uniform at every pixel. The alpha and offset map for each projector is generated by cutting off the appropriate region from the respective maps for the display using the geometric calibration information (Figure 7).
In $[20,18]$, a linear function that does not approximate the response of the eye appropriately was chosen as the uniform gamma function, $G$. This led to washed out images. We use $G(i)=i^{2}$ to approximate the logarithmic response of the human eye to varying brightness. Also, note that $G^{-1}$ varies from projector to projector and is given by $g^{-1}$, i.e. the inverse of the gamma function of each projector. To measure $g$ for each projector we use the method proposed in [22]. $g^{-1}$ is then generated from $g$ and represented by a look-up-table.

Thus, the final correction is achieved by applying the following three steps to the input image of each projector.

1. Multiply the image by itself to apply the uniform gamma function.

2.Multiply the resulting image by the projector alpha map and add the projector offset map.

3.Apply a inverse of the gamma function to the resultant image via a look up table.

All the above steps are applied in real-time using the pixel shaders of commodity graphics hardware. The scene is first rendered to texture and then the corrections are applied. Multi-texturing is used for applying the uniform gamma function and the alpha and offset maps. Choosing the uniform gamma function to be $i^{2}$, as opposed to $i^{1.8}$ or $i^{2.2}$ (as used in Macintosh or Windows) helps us to implement this step in the pixel shader using multi-texturing as a multiplication of the image by itself. The inverse gamma function is applied using dependent 2D texture look-ups. This is followed by an image warp to correct the geometric misalignments using [10].

\subsection{Color Multi-Display}

The obvious way to extend this method to color is to apply the same method to the three channels independently, i.e. use the brightness smoothing method to smooth the $H_{D}$ for each channel. However, the concern is to maintain a spatially uniform white balance across the display thus avoiding introduction of color blotches.

The white balance at any pixel $(x, y)$ of the display depends on the proportion of the $H_{D}$ from each channel with respect to the other two. In fact, it can be shown that if the shape of $H_{D}$ remains the same across different channels, it assures that the proportions of red, green and blue at 


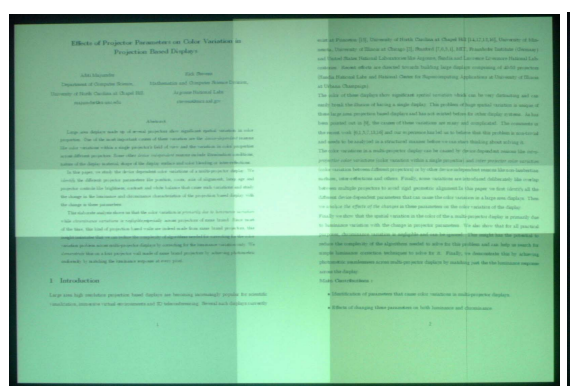

(a)

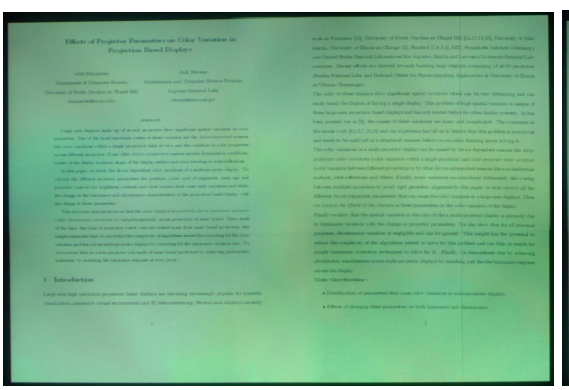

(b)

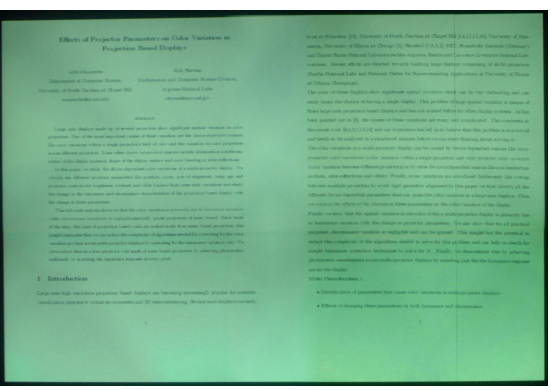

(c)

Figure 8: Digital photographs of a four projector tiled display $\left(1.5^{\prime} \times 2.5^{\prime}\right.$ in size) before any correction (a), after applying brightness smoothing independently to the three color channel that does not assure spatial constancy in white balance leading to color blotches $(b)$, after applying the brightness smoothing to the green channel and duplicating the alpha map of the green channel for the red and blue channel that removes the color blotches $(c)$. Note that the blotches remaining in (c) is also present in (a) before correction and are due to spatial variation in chrominance and hence cannot be fixed by the brightness modification alone. (Please see in color)

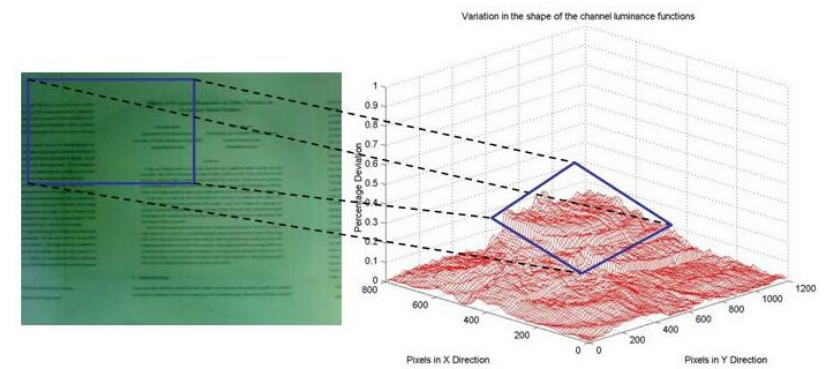

Figure 9: Left: Color blotches on a single projector. Right: Corresponding percentage deviation in the shape of the $\widehat{H}^{g}$ and $\widehat{H}^{b}$ (the maximum deviation is $20 \%$ and the visible color blotches corresponded with the regions of large deviation).

every pixel is identical and hence white balance is uniform across the display. Let $H_{D}^{l}$ denote the $H_{D}$ for channel $l$, $l \in\{r, g, b\}$. The shape of $H_{D}^{l}$, denoted by $\widehat{H}_{D}^{l}$, is given by normalizing it, i.e.,

$$
H_{D}^{l}(x, y)=l_{\max } \cdot \widehat{H}_{D}^{l}(x, y)
$$

where $l_{\max }=\max _{\forall(x, y)} H_{D}^{l}$. Now, if $\widehat{H}_{D}^{l}$ is identical for all $l$, the proportion of contribution from channel $l$ at pixel $(x, y), P_{l}(x, y)$, is given by

$$
P_{l}(x, y)=\frac{H_{D}^{l}(x, y)}{\sum_{l \in\{r, g, b\}} H_{D}^{l}(x, y)}=\frac{l_{\max }}{\sum_{l \in\{r, g, b\}} l_{\max }},
$$

and is independent of $(x, y)$, i.e. white balance is spatially uniform.

This can be verified empirically for single projectors which often show color blotches. Though some of these blotches correspond to spatial variation in chrominance properties, some are also caused by the difference in the shape of $\widehat{H}^{l}$ across different channels. Using Equation 15, we reconstructed $\widehat{H}^{l}, l \in\{r, g, b\}$ for stand-alone projectors and found that many of the color blotches occur in the spatial region where the $\widehat{H}^{l}$ across different channels deviate from each other (Figure 9).

Also, studying the similarity of $\widehat{H}_{D}^{l}$ across different channels in multi-projector displays empirically, we found the maximum deviation to be less than $20 \%$, resulting in color blotches. Applying the method described in Section 3.1 independently to each channel does not assure identical shape of $H_{D}^{\prime}$ across different channels, and hence can introduce additional color blotches. To avoid this, we apply the smoothing to the $\widehat{H}_{D}^{l}$ of any one channel and then use the alpha map generated from this channel across all channels. Thus, the alpha map generated for each channel of our correction is identical. Note that this assures that even if $\widehat{H}_{D}^{l}$ is not identical across different channels before brightness smoothing, their shapes are similar after the correction is applied and hence a spatially constant white balance is achieved (Figure 8). However, this only corrects the color blotches due to variation in $\widehat{H}^{l}$ and cannot fix the ones due to spatial variation in chrominance.

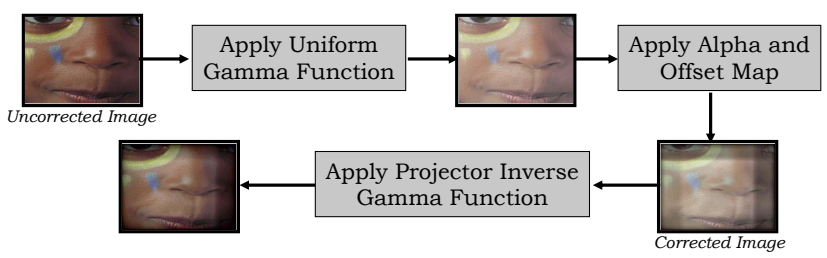

Figure 10: Per Projector Correction

Since $L_{D}$ and $L_{D}^{\prime}$ are independent of the number of channels, the offset map of the display generated in case of color displays is same as the gray display. To distribute this map to the three channels while correction, the offset map is divided by 3 . The final real-time online correction is illustrated in Figure 10 and details of the implementation are available at [1].

\subsubsection{Results}

We demonstrated our method successfully on three different display systems, made of $2 \times 2$ array of four projectors $\left(1.5^{\prime} \times 2.5^{\prime}\right), 2 \times 3$ array of six projectors $\left(3^{\prime} \times 4^{\prime}\right)$ and $3 \times 5$ array of fifteen projectors $\left(8^{\prime} \times 10^{\prime}\right)$ (Figure 12 and 13).

Since our method assumes view-independent or Lambertian screens, our correction is accurate from the position of 


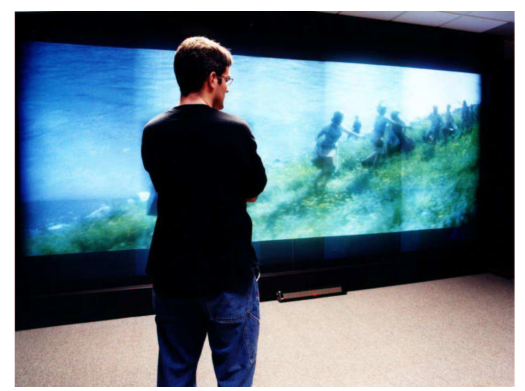

Figure 11: A non-Lambertian display made of $3 \times 5$ array of fifteen projectors viewed from a view direction that makes an angle of less than 20 degrees with the screen.

the camera used for capturing $H_{D}$ and $L_{D}$. However, in practice our result looks seamless for a wide range of viewing angles and distances from the display. For our jenmar screen with gain of approximately 2.0 , we see no artifacts if the view direction makes an angle of about $20-90$ degrees with the plane of the screen. For less than 20 degrees, the boundaries of the projectors are visible, but as smooth edges, to some extent like the result of an edge-blending method (Figure 11).

We derive the contrast parameter, $\lambda$, as follows. Let $d$ be the perpendicular distance of the user (camera) from the display, $r$ be the resolution of the display in pixels per unit distance and $\tau$ be the brightness threshold that humans can tolerate per degree of visual angle. From CSF, $\tau$ is $1 \%$ at the frequency of peak sensitivity, 5 cycles/degree. The number of display pixels subtended per cycle of the grating at this sensitivity is given by $\frac{d \pi r}{180 \times 5}$. A brightness variation of $\tau$ within these pixels will go undetected by humans and hence, $\lambda=\frac{900 \tau}{d \pi r}$. For our fifteen projector display with $r=30, d=6$ and $\tau=0.01$, we get a $\lambda$ of 0.00125 . Note that as the user moves farther away from the display $\lambda$ goes up, i.e. the surface needs to be smoother and hence will have lower contrast. This explains the variations which are not perceptible on a large display being perceptible in some of our results in Figure 12, and 13. The results in the paper being highly scaled down images of the display, simulate a situation where $d=\infty$ and hence $\lambda=\infty$ is required to make these variations imperceptible in paper.

\section{Conclusion}

In conclusion, we have presented a method that uses human contrast sensitivity function to achieve brightness seamlessness in multi-displays. In a periodic calibration phase, a digital camera is used to capture the brightness response of the display which is modified optimally to maximize the display contrast while minimizing noticeable brightness variations. An alpha and offset map for each projector generated from this modified response is then used to change the input images in real-time on commodity graphics hardware to correct for the brightness variation. The result is a highcontrast seamless multi-display. This efficient and scalable
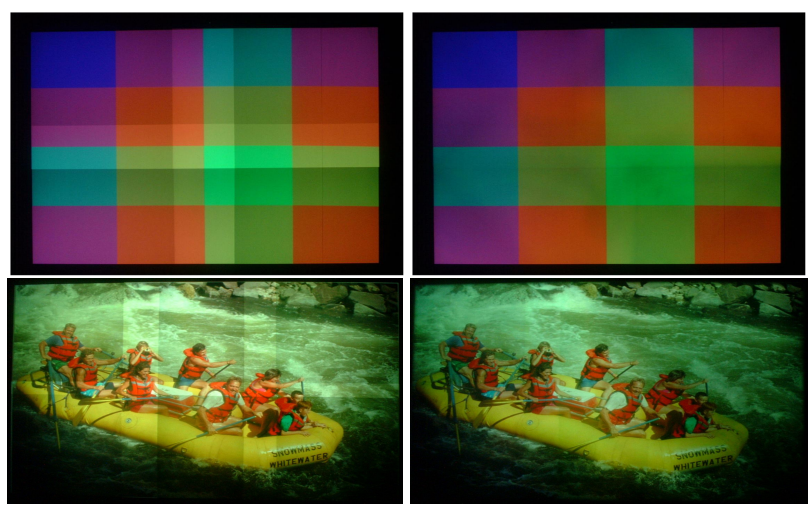

Figure 12: Digital photographs of displays made of $2 \times 2$ of four (top) and $2 \times 3$ array of six (bottom) projectors of size $1.5^{\prime} \times 2.5^{\prime}$ and $3^{\prime} \times 4^{\prime}$ respectively. Left: Before correction. Right: After brightness smoothing. Note that we are able to achieve seamlessness even for flat colors, the most critical test for our algorithm.

method has been demonstrated in many multi-displays of different size and resolution.

However, we believe that our work is just the first step towards solving the more general problem of color seamlessness in multi-projector displays. We do the best that is possible while considering only brightness assuming that all projectors have identical red, green and blue chromaticity (which is not true in practice). However, we can envision devising a $5 \mathrm{D}$ optimization method that considers the gamut of each projector while smoothing the 5D color response (one parameter for brightness, two parameters for chrominance and two parameters for spatial coordinates). Finally, to enable different defense applications, self-calibrating systems that can correct themselves in realtime from arbitrary images projected on the display needs to be devised.

\section{References}

[1] J. Binns, G. Gill, M. Hereld, D. Jones, I. Judson, T. Leggett, A. Majumder, M. McCroy, M. E. Papka, and R. Stevens. Applying geometry and color correction to tiled display walls (poster). IEEE Visualization, 2002.

[2] Ian Buck, Greg Humphreys, and Pat Hanrahan. Tracking graphics state for networked rendering. Proceedings of Eurographics/SIGGRAPH Workshop on Graphics Hardware, pages 87-95, 2000.

[3] A. Cazes, G. Braudaway, J. Christensen, M. Cordes, D. DeCain, A. Lien, F. Mintzer, and S. L. Wright. On the color calibration of liquid crystal displays. SPIE Conference on Display Metrology, pages 154-161, 1999.

[4] C. J. Chen and Mike Johnson. Fundamentals of scalable high resolution seamlessly tiled projection system. Proceedings of SPIE Projection Displays VII, 4294:67-74, 2001.

[5] Han Chen, Rahul Sukthankar, Grant Wallace, and Kai Li. Scalable alignment of large-format multi-projector displays using camera homography trees. Proceedings of IEEE Visualization, pages 339-346, 2002. 

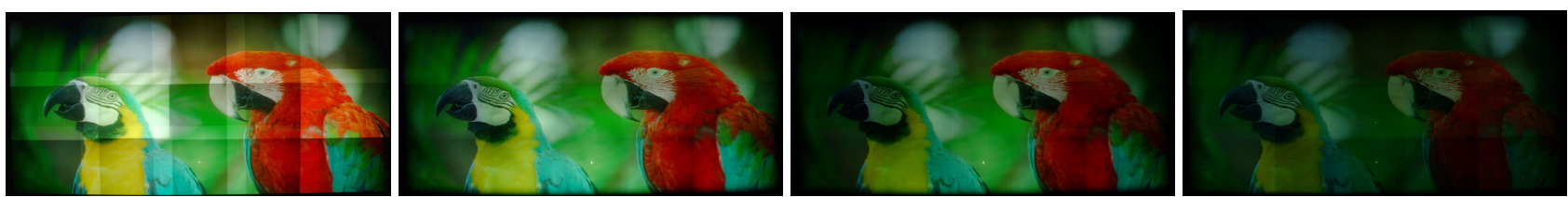

Figure 13: Digital photographs of a fifteen projector tiled display $\left(8^{\prime} \times 10^{\prime}\right.$ in size). From left: Before any correction, after brightness smoothing with contrast parameter of $\lambda=0.0025$, for $\lambda=0.00125$ and after brightness uniformity i.e. $\lambda=0$. Note that the contrast of the display reduces as $\lambda$ decreases. At $\lambda=0$ is the special case of brightness uniformity where the display has a very low contrast.

[6] R.A. Chorley and J. Laylock. Human factor consideration for the interface between electro-optical display and the human visual system. In Displays, volume 4, 1981.

[7] Carolina Cruz-Neira, Daniel J. Sandin, and Thomas A.Defanti. Surround-screen projection-based virtual reality: The design and implementation of the CAVE. In Proceedings of ACM SIGGRAPH, pages 135-142, 1993.

[8] E. Bruce Goldstein. Sensation and Perception. Wadsworth Publishing Company, 2001.

[9] Rafael C. Gonzalez and Richard E. Woods. Digital Image Processing. Addison Wesley, 1992.

[10] Mark Hereld, Ivan R. Judson, and Rick Stevens. Dottytoto: A measurement engine for aligning multi-projector display systems. Argonne National Laboratory preprint ANL/MCSP958-0502, 2002.

[11] G. Humphreys, M. Eldridge, I. Buck, G. Stoll, M. Everett, and P. Hanrahan. Wiregl: A scalable graphics system for clusters. Proceedings of ACM SIGGRAPH, pages 129-140, 2001.

[12] G. Humphreys and P. Hanrahan. A distributed graphics system for large tiled displays. In Proceedings of IEEE Visualization, pages 215-223, 1999.

[13] Greg Humphreys, Ian Buck, Matthew Eldridge, and Pat Hanrahan. Distributed rendering for scalable displays. Proceedings of IEEE Supercomputing, 2000.

[14] E.H. Land and J.J. McCann. Lightness and retinex theory. Journal of Optical Society of America, 61(1):1-11, 1971.

[15] K. Li, H. Chen, Y. Chen, D. W. Clark, P. Cook, S. Damianakis, G. Essl, A. Finkelstein, T. Funkhouser, A. Klein, Z. Liu, E. Praun, R. Samanta, B. Shedd, J. P. Singh, G. Tzanetakis, and J. Zheng. Early experiences and challenges in building and using a scalable display wall system. IEEE Computer Graphics and Applications, 20(4):671-680, 2000.

[16] Aditi Majumder. Properties of color variation across multiprojector displays. Proceedings of SID Eurodisplay, pages 807-810, 2002.

[17] Aditi Majumder, Zue He, Herman Towles, and Greg Welch. Achieving color uniformity across multi-projector displays. Proceedings of IEEE Visualization, pages 117-124, 2000.

[18] Aditi Majumder, David Jones, Matthew McCrory, Michael E. Papka, and Rick Stevens. Using a camera to capture and correct spatial photometric variation in multi-projector displays. IEEE International Workshop on Projector-Camera Systems, 2003.
[19] Aditi Majumder and Rick Stevens. LAM: Luminance attenuation map for photometric uniformity in projection based displays. Proceedings of ACM Virtual Reality and Software Technology, pages 147-154, 2002.

[20] Aditi Majumder and Rick Stevens. Color nonuniformity in projection-based displays: Analysis and solutions. IEEE Transactions on Visualization and Computer Graphics, 10(2):177-188, March/April 2004.

[21] B. Pailthorpe, N. Bordes, W.P. Bleha, S. Reinsch, and J. Moreland. High-resolution display with uniform illumination. Proceedings Asia Display IDW, pages 1295-1298, 2001.

[22] Andrew Raij, Gennette Gill, Aditi Majumder, Herman Towles, and Henry Fuchs. Pixelflex2: A comprehensive, automatic, casually-aligned multi-projector display. IEEE International Workshop on Projector-Camera Systems, 2003.

[23] R. Raskar, M.S. Brown, R. Yang, W. Chen, H. Towles, B. Seales, and H. Fuchs. Multi projector displays using camera based registration. Proceedings of IEEE Visualization, pages 161-168, 1999.

[24] R. Raskar, G. Welch, M. Cutts, A. Lake, L. Stesin, and H. Fuchs. The office of the future: A unified approach to image based modeling and spatially immersive display. In Proceedings of ACM SIGGRAPH, pages 168-176, 1998.

[25] Ramesh Raskar. Immersive planar displays using roughly aligned projectors. In Proceedings of IEEE Virtual Reality 2000, pages 109-116, 1999.

[26] Rudro Samanta, Jiannan Zheng, Thomas Funkhouse, Kai Li, and Jaswinder Pal Singh. Load balancing for multi-projector rendering systems. In SIGGRAPH/Eurographics Workshop on Graphics Hardware, pages 107-116, 1999.

[27] Maureen C. Stone. Color and brightness appearance issues in tiled displays. IEEE Computer Graphics and Applications, pages 58-66, 2001.

[28] Maureen C. Stone. Color balancing experimental projection displays. 9th IS\&T/SID Color Imaging Conference, pages 342-347, 2001.

[29] Russell L. De Valois and Karen K. De Valois. Spatial Vision. Oxford University Press, 1990.

[30] Grant Wallace, Han Chen, and Kai Li. Color gamut matching for tiled display walls. Proceedings of Immersive Projection Technology Symposium (IPT), 2003.

[31] Ruigang Yang, David Gotz, Justin Hensley, Herman Towles, and Michael S. Brown. Pixelflex: A reconfigurable multiprojector display system. Proceedings of IEEE Visualization, pages 167-174, 2001. 